\title{
Determining Longwave Forcing and Feedback Using Infrared Spectra and GNSS Radio Occultation
}

\author{
Yi Huang, Stephen S. Leroy, And James G. Anderson \\ Department of Earth and Planetary Sciences and the School of Engineering and Applied Sciences, \\ Harvard University, Cambridge, Massachusetts
}

(Manuscript received 22 December 2009, in final form 11 May 2010)

\begin{abstract}
The authors investigate whether combining a data type derived from radio occultation (RO) with the infrared spectral data in an optimal detection method improves the quantification of longwave radiative forcing and feedback. Signals derived from a doubled- $\mathrm{CO}_{2}$ experiment in a theoretical study are used. When the uncertainties in both data types are conservatively estimated, jointly detecting the feedbacks of tropospheric temperature and water vapor, stratospheric temperature, and high-level cloud from the two data types should reduce the mean errors by more than $50 \%$. This improvement is achieved because the RO measurement helps disentangle the radiance signals that are ambiguous in the infrared spectrum. The result signifies the complementary information content in infrared spectral and radio occultation data types, which can be effectively combined in optimal detection to accurately quantify the longwave radiative forcing and feedback. The results herein show that the radiative forcing of $\mathrm{CO}_{2}$ and the longwave radiative feedbacks of tropospheric temperature, tropospheric water vapor, and stratospheric temperature can be accurately quantified from the combined data types, with relative errors in their global mean values being less than $4 \%$, $10 \%, 15 \%$, and $20 \%$, respectively.
\end{abstract}

\section{Introduction}

Climatic feedbacks of atmospheric temperature, water vapor, cloud, etc., as measured by their impacts on the topof-the-atmosphere (TOA) radiation fluxes, are currently not well constrained by observations and differ considerably in climate models (Bony et al. 2006). Leroy et al. (2008) and Huang et al. (2010) used an optimal detection (OD) method (multivariate linear regression) to determine longwave climate feedbacks from spectral measurements. This method takes advantage of the infrared (IR) spectral features of climate forcings of greenhouse gases and feedbacks of temperature, water vapor, and clouds and quantifies each of them by partitioning the total change in the outgoing longwave radiation (OLR) spectrum. One limitation of this method when applied to IR spectral measurement, however, is the ambiguity issue. Some feedbacks have similar infrared spectral fingerprints: when their fingerprints are obscured by uncertainties, it becomes difficult to quantify them. Particularly,

Corresponding author address: Yi Huang, 12 Oxford St., Link 284, Cambridge, MA 02138.

E-mail: yihuang@huarp.harvard.edu
Huang et al. (2010) noted the fingerprint ambiguities between tropospheric temperature and water vapor, between tropospheric temperature and high-level cloud, and between surface temperature and low-level cloud.

Incorporating another set of satellite measurements that better distinguishes signals similar in the thermal infrared spectrum provides a potential solution to the difficulty. Like spectrally resolved infrared radiances, the quantities (phase delay in the transmitted radio signal, satellite position, velocity, etc.) in the Global Navigation Satellite Systems (GNSS) radio occultation (RO) needed to derive atmospheric microwave refractivity profiles can be measured with high accuracy in space (Goody et al. 1998; Kursinski et al. 1997). With the measurement uncertainties well constrained and precisely quantified, accumulated IR spectral and GNSS RO data will describe various subtle changes in the climate system and form valuable climatic records.

However, every remote sensing measurement is based on a particular inverse method that relates the directly measured quantity to a geophysical variable of interest. Combining two arbitrary measurements does not guarantee an improvement in determining a variable because their information content may not be complementary. 
Moreover, in studying long-term climate trend signals, it is very difficult, if not impossible, to trace the uncertainty in an operationally retrieved geophysical variable to measurement errors or instrument change because of the complexity of retrieval algorithms. Optimal detection, however, which applies to direct measurements and links the changes in them to climate forcing and feedbacks through a linear regression, affords a simple way to trace the propagation of errors and quantify the overall uncertainty (Huang et al. 2010). Building upon the work of Huang et al. (2010), the purpose of this paper is to investigate whether incorporating a GNSS RO observable into optimal detection reduces the uncertainty in detection results obtained with the IR spectrum alone. In comparison with the IR spectrum, the GNSS RO active microwave measurement is sensitive to variations in temperature and water vapor but is insensitive to clouds. So we are particularly interested in whether there is an improvement in temperature and water vapor longwave feedbacks and whether the evaluation of cloud feedback can benefit as well. In this theoretical study, we ignore the instrumentation-related uncertainties in actual datasets; rather, we focus on understanding the uncertainties arising from the inverse method itself when jointly applied to IR spectra and GNSS RO data.

Following Huang et al. (2010), we generate hypothetical climate change signals in IR spectra and RO logdry pressure profiles through a forward simulation based on a general circulation model (GCM) doubled- $\mathrm{CO}_{2}$ experiment. In section 2, we describe the GCM experiment and the spectral and RO simulations. In section 3, we investigate the joint optimal detection with the two data types. In section 4 , we summarize and discuss the benefits and limitations of incorporating refractivity measurement.

\section{Simulations}

As detailed by Huang et al. (2010), we use the difference between the beginning and ending steady states in a doubled- $\mathrm{CO}_{2}(280-560 \mathrm{ppmv})$ experiment by CCCMA, an atmosphere-slab ocean coupled GCM participating in the Cloud Feedback Model Intercomparison Project (CFMIP; Williams and Webb 2009), as a surrogate for climate change. CFMIP data are chosen because the dataset includes 3D fields of cloud properties that are necessary for computing IR spectra. The model-generated monthly mean atmospheric profiles of temperature, specific humidity, and cloud fraction and condensate concentration are archived at 17 pressure levels with $2.5^{\circ} \times$ $2.5^{\circ}$ horizontal resolution. We have used the 20 years of the steady state prior to a step $\mathrm{CO}_{2}$ doubling and 15 years of the post-doubling steady state to simulate spectral IR radiances and RO log-dry pressure.

IR spectra ranging from 100 to $3300 \mathrm{~cm}^{-1}$ are simulated using a moderate-resolution radiative transfer model, MODTRAN 4 (Bernstein et al. 1996), at 2- $\mathrm{cm}^{-1}$ resolution. Five additional layers of standard atmosphere (McClatchey et al. 1972) are patched to the top of the 17-layer profiles in order to properly represent the stratospheric absorbers. Clouds are assumed to overlap randomly for the all-sky computation. Except for water vapor and $\mathrm{CO}_{2}$, other greenhouse gases including $\mathrm{O}_{3}, \mathrm{CH}_{4}, \mathrm{~N}_{2} \mathrm{O}$, and chlorofluorocarbons (CFCs) are prescribed with constant values. More details of the spectral radiance simulation are described by Huang et al. (2007a).

Microwave refractivity $(N)$ is computed using a widely used empirical formula

$$
N=a \frac{P}{T}+b \frac{P_{W}}{T^{2}},
$$

where $T, P$, and $P_{W}$ are atmospheric temperature, pressure, and partial pressure of water vapor, respectively. The constants $a=77.6 \mathrm{~K} \mathrm{hPa}^{-1}$ and $b=373 \times 10^{3} \mathrm{~K}^{2} \mathrm{hPa}^{-1}$ are empirically determined (Smith and Weintraub 1953). The refractivity uncertainty caused by parameter uncertainty usually is far less than 1\% (Kursinski et al. 1997) and is thus negligible compared to the uncertainty level of $5 \%-20 \%$ due to signal shape uncertainty and other causes considered in the analysis below. Following Leroy and North (2000) and Leroy et al. (2006), we use the logarithm of the integrated refractivity- "dry pressure" $\left(P_{d}\right)$, whose physical meaning is easy to interpret-to construct the feedback fingerprints. By definition,

$$
P_{d}(h)=\frac{\mu_{d} g}{a R} \int_{h}^{\infty} N\left(h^{\prime}\right) d h^{\prime},
$$

where $\mu_{d}$ is the molecular mass of dry air, $g$ is the gravity constant $\left(9.80665 \mathrm{~m} \mathrm{~s}^{-2}\right), R$ is the ideal gas constant, and $h$ and $h^{\prime}$ are height coordinates. Replacing $N$ with Eq. (1), applying the hydrostatic equation and the ideal gas law, and approximating the mean molecular mass to be $\mu_{d}$, we obtain

$$
P_{d}(h)=P(h)+\frac{b}{a} \frac{\mu_{d}}{\mu_{w}} \int_{0}^{P(h)} \frac{q\left(P^{\prime}\right)}{T\left(P^{\prime}\right)} d P^{\prime},
$$

where $\mu_{w}$ is the molecular mass of water vapor and $q$ is specific humidity. The impact of atmospheric water vapor is given by the second term on the right-hand side. In the upper atmosphere where the water vapor content is negligible, $P_{d}$ is close to $P$, and variations in $\ln \left(P_{d}\right)$ are strongly related to variations in geopotential height 
according to the hydrostatic equation. Because height, instead of pressure, is the natural independent vertical coordinate for RO retrieval (Leroy 1997), we first compute $P_{d}$ from CCCMA profiles with Eq. (3) and then interpolate $\ln \left(P_{d}\right)$ into 17 fixed height levels: $0,0.8,1.5,3$, $4,5.5,7,9,10,12,14,16,18,20,23,27$, and $30 \mathrm{~km}$.

\section{Optimal detection}

Optimal detection (Bell 1986; North et al. 1995; Hasselmann 1997) is a multipattern linear regression:

$$
\delta \mathbf{y}=\mathbf{S a}+\mathbf{r} .
$$

The column vector $\delta \mathbf{y}\left[n_{s} \times 1\right]$ contains the total change signals in the measurement. In general, the observation vector $\mathbf{y}$ can contain any combination of data types. In this study, $\mathbf{y}$ contains all the spectral channels of the IR spectrum and all the levels of log-dry pressure profiles. The columns of the matrix $\mathbf{S}\left[n_{s} \times n_{f}\right]$ contain the fingerprints of the $n_{f}$ physical contributors $\left(X_{i}, i=1, \ldots, n_{f}\right)$. The signal amplitude vector $\mathbf{a}\left[n_{f} \times 1\right]$ is the solution to the problem we seek - the amplitude of each of the $n_{f}$ contributors that collectively give rise to the total change signals. The vector $\mathbf{r}\left[n_{s} \times 1\right]$ is the residual that cannot be explained by linear combination of the fingerprints. The least mean squares solution to Eq. (4) is

$$
\mathbf{a}_{\mathrm{ml}}=\left(\mathbf{S}^{\mathrm{T}} \boldsymbol{\Sigma}^{-1} \mathbf{S}\right)^{-1} \mathbf{S}^{\mathrm{T}} \boldsymbol{\Sigma}^{-1} \delta \mathbf{y},
$$

where $\boldsymbol{\Sigma}$ is the total uncertainty covariance matrix. The rows of $\left(\mathbf{S}^{\mathrm{T}} \boldsymbol{\Sigma}^{-1} \mathbf{S}\right)^{-1} \mathbf{S}^{\mathrm{T}} \mathbf{\Sigma}^{-1}$ are the "optimal fingerprints" (Leroy and Anderson 2010); applying them to data, one obtains $\mathbf{a}_{\mathrm{ml}}$, the most likely fit of $\mathbf{a}$ to the data. The matrix inverse $\boldsymbol{\Sigma}^{-1}$ is computed with a matrix pseudoinversion: $\boldsymbol{\Sigma}^{-1}=\mathbf{e} \boldsymbol{\lambda}^{-1} \mathbf{e}^{\mathrm{T}}$, where the columns of $\mathbf{e}\left[n_{s} \times\right.$ $n_{e}$ ] are the first $n_{e}$ eigenvectors of $\boldsymbol{\Sigma}$ and the diagonal of $\boldsymbol{\lambda}\left[n_{e} \times n_{e}\right]$ contains the corresponding eigenvalues. (Offdiagonal elements of $\boldsymbol{\lambda}$ are zero.) In this study, we retain the first 50 eigenvectors and eigenvalues $\left(n_{e}=50\right)$. The uncertainties of individual elements of $\mathbf{a}_{\mathrm{ml}}$ are the square roots of the diagonal elements of $\left(\mathbf{S}^{\mathrm{T}} \mathbf{\Sigma}^{-1} \mathbf{S}\right)^{-1}$.

The total uncertainty matrix is $\mathbf{\Sigma}=\mathbf{\Sigma}_{v}+\mathbf{\Sigma}_{u}+\mathbf{\Sigma}_{\mathrm{nl}}$, where the terms on the right-hand side are the contributions of natural variability, uncertainty in the fingerprints, and overall nonlinearity, respectively. The natural variability covariance is computed from an unperturbed run of the climate model

$$
\Sigma_{v}=\operatorname{cov}(\mathbf{y})
$$

the fingerprint uncertainty is computed by

$$
\mathbf{\Sigma}_{u}=\left\langle\sum_{i, j}^{n_{f}}\left(\delta \mathbf{y}_{i}^{N}-\left\langle\delta \mathbf{y}_{i}^{N}\right\rangle\right)\left(\delta \mathbf{y}_{j}^{N}-\left\langle\delta \mathbf{y}_{j}^{N}\right\rangle\right)^{\mathrm{T}}\left\langle\delta F_{i}\right\rangle\left\langle\delta F_{j}\right\rangle\right\rangle,
$$

and the nonlinearity is computed by

$$
\Sigma_{\mathrm{nl}}=\left\langle\left(\delta \mathbf{y}_{\text {total }}-\sum_{i} \delta \mathbf{y}_{i}\right)\left(\delta \mathbf{y}_{\text {total }}-\sum_{i} \delta \mathbf{y}_{i}\right)^{\mathrm{T}}\right\rangle .
$$

The vector $\delta \mathbf{y}_{\text {total }}$ denotes a single realization of the total change signal; $\delta \mathbf{y}_{i}$, the partial change signal contributed by $X_{i} ; \delta F_{i}$, the magnitude of radiative forcing or feedback by $X_{i}$, measured by OLR broadband flux change; and $\delta \mathbf{y}_{i}^{N}=\delta \mathbf{y}_{i} / \delta F_{i}$, the fingerprint of the $i$ th forcing or feedback corresponding to change in $X_{i}$. With this normalization, the $i$ th element of $\mathbf{a}$ and $\mathbf{a}_{\mathrm{ml}}$ is an estimate of $\delta F_{i}$.

In Eqs. (7) and (8), $\langle\cdots\rangle$ denotes an average over individual realizations of climate change, which, in this study, are all the individual grid point elements of CCCMA. Here, spatial variation is used to represent the impact of uncertain physics of climate change on the fingerprints because we are limited by the availability of $3 \mathrm{D}$ cloud fields from either a multimodel ensemble or a perturbed physics ensemble (Huang et al. 2010).

We focus on the all-sky application and use the numbers given by Huang et al. (2010) for the reference values ("truth") of the $\mathrm{CO}_{2}$ forcing and longwave feedbacks $\left(\delta F_{i}\right.$; see Huang et al's Table 1); we also refer the readers to the results there (their Fig. 2) for the IR spectral fingerprints, $\delta R_{i}^{N}$. These results are obtained by using the partial radiation perturbation (PRP) method (Wetherald and Manabe 1988). In this paper, we compute the RO fingerprints with the same perturbation idea. First, two sets of dry pressure profiles $\left(P_{d}^{1}\right.$ and $\left.P_{d}^{2}\right)$ corresponding to the two steady periods of the doubled- $\mathrm{CO}_{2}$ experiment are computed. The total change signal is obtained as the difference between the logarithms of two period means: $\left(\delta \ln P_{d}\right)_{\text {total }}=\ln \overline{P_{d}^{2}}-\ln \overline{P_{d}^{1}}$, with the overbars indicating temporal means. Then, we replace the values of a variable $X_{i}$ in the ending period with its mean value in the beginning period and recalculate another set of dry pressure profile $\left(P_{d}^{2}\right)_{i}$. The change in the dry pressure profile contributed by $X_{i}$ is computed as $\left(\delta \ln P_{d}\right)_{i}=$ $\ln \left(\overline{P_{d}^{2}}\right)-\ln \left(\overline{P_{d}^{2}}\right)_{i}$. Because the phase delay in RO signals is insensitive to cloud (its contribution to refractivity, even in the case of thick and extended cumulus, is unlikely to exceed $1 \%$ ), we have considered only the noncloud contributors. These contributors include tropospheric temperature, tropospheric water vapor, stratospheric temperature, and stratospheric water vapor. For convenience, 


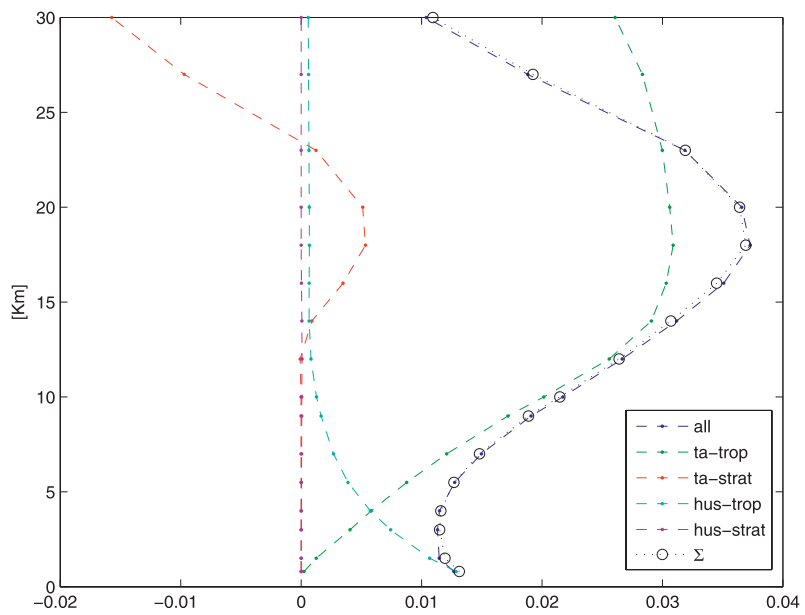

FIG. 1. Changes in global mean dry pressure profile contributed by tropospheric temperature (denoted "ta-trop"), stratospheric temperature ("ta-strat"), tropospheric water vapor ("hus-trop"), and stratospheric water vapor ("hus-strat"). The overall change ("all") when considering all the temperature and water vapor changes simultaneously, $\left(\delta \ln P_{d}\right)_{\text {total }}$, is compared to the sum $\left(\sum\right)$ of individual contributions, $\sum_{i}\left(\delta \ln P_{d}\right)_{i}$.

the tropopause is set to be $200 \mathrm{hPa}$ globally [see Huang et al. (2010) for a discussion of the caveat].

Figure 1 illustrates the global mean log-dry pressure profile change $\left\langle\left(\delta \ln P_{d}\right)_{i}\right\rangle$ due to the different physical contributors. The overall change signal obtained considering all the contributors together is well reproduced by the sum of individual contributions. This indicates that logdry pressure can be linearly partitioned to a great extent. Stratospheric water vapor contributes negligibly, which results from the very small water vapor content in the stratosphere. The other three contributors-tropospheric temperature, tropospheric water vapor, and stratospheric temperature-result in very different changes in the logdry pressure profile. In the doubled- $\mathrm{CO}_{2}$ experiment, the troposphere generally warms in response to the $\mathrm{CO}_{2}$ forcing. Under the hydrostatic balance $d P / P=-(g / R T) d h$, the increase of temperature makes a fixed-pressure layer thicker. This equivalently increases pressure and thus logdry pressure at every given height level. The impact of the tropospheric warming integrates with height and maximizes at the lower stratosphere where temperature change changes the sign. In contrast, the stratosphere generally cools in response to the $\mathrm{CO}_{2}$ forcing, which reduces dry pressure. The moistening in the troposphere increases dry pressure; this signal is the most significant in the lower troposphere because water vapor concentrations are greatest there. While the "wet-dry ambiguity" in radio occultation exists in retrieval, the wet (water vapor) and dry (temperature) terms are distinguished here because the vertical structure of their changes (column-wise warming and moistening) are taken into account. The contrast in shapes provides the basis for including log-dry pressure in the fingerprints for the total change signal.

Fingerprinting longwave radiative forcing and feedbacks $\left(\delta F_{i}\right)$ in the IR spectrum $\left(\delta R_{\text {total }}\right)$ is motivated by the fact that $\delta F_{i}$ is characterized by different partial radiance change patterns $\left(\delta R_{i}\right)$, as recognized by many previous authors (Kiehl 1983; Slingo and Webb 1997; Haskins et al. 1999; Huang et al. 2007b; Leroy et al. 2008; Huang and Ramaswamy 2009). Optimal detection requires an accounting for how the uncertainty in fingerprints contributes to the uncertainty in the interpreted forcing and feedbacks (Huang et al. 2010; Leroy and Anderson 2010). This accounting is made by normalizing partial radiance $\delta R_{i}$ by associated OLR changes and then computing the variation of the normalized spectral fingerprints $\delta R_{i}^{N}$. If the unnormalized $\delta R_{i}$ contains insufficient information to determine $\delta F_{i}$, then the normalized $\delta R_{i}^{N}$ will have disparate shapes and $\boldsymbol{\Sigma}_{u}$ would be inflated, leading to overly large uncertainty in OD results.

Extending the problem to include RO log-dry pressure demands the same normalization if the forcing and feedbacks are still assessed with radiative flux changes. When the perturbation is small, $\delta F_{i}$ and $\left(\delta \ln P_{d}\right)_{i}$, to the first-order approximation, are both proportional to the change in the controlling geophysical variable, $\delta X_{i}$. If the assumption of linearity remains valid, $\left(\delta \ln P_{d}\right)_{i}$ should be a strong predictor of $\delta F_{i}$. We check that refractivity measurement can be linked to feedback values by correlating the column-average dry pressure change $\left[\int_{0}^{h_{\text {top }}}\left(\delta \ln P_{d}\right)_{i} d h\right] / h_{\text {top }}\left(h_{\text {top }}=30 \mathrm{~km}\right)$ to $\delta F_{i}$. The correlation coefficients over all the CCCMA grid points across the globe are $0.87,0.88,-0.82$, and -0.93 for tropospheric temperature, stratospheric temperature, tropospheric water vapor, and stratospheric water vapor, respectively. The correlations are strong enough to justify normalizing the dry pressure signals by the longwave radiation flux and using $\left(\delta \ln P_{d}\right)_{i}^{N}=\left(\delta \ln P_{d}\right)_{i} / \delta F_{i}$ as part of the fingerprint vector.

We assess the uncertainty in the optimally detected feedback results with two tests. First, we develop a set of optimal fingerprints using normalized global mean partial change signals, forming the $i$ th column of $\mathbf{S}, \mathbf{s}_{i}=$ $\left[\left\langle\delta R_{i}\right\rangle /\left\langle\delta F_{i}\right\rangle,\left\langle\left(\delta \ln P_{d}\right)_{i}\right\rangle /\left\langle\delta F_{i}\right\rangle\right]$, and apply the optimal fingerprints to the global mean total change in the IR spectrum and log-dry pressure profile $\left[\left\langle\delta R_{\text {total }}\right\rangle,\left\langle\delta \ln P_{d \text {,total }}\right\rangle\right]$. Table 1 shows the OLR changes induced by five contributors determined in this way. Because we have used accurate fingerprints in obtaining the OD results, the difference between the most likely $a_{\mathrm{ml}}$ and "truth" does not reflect the uncertainty whereas the numbers determined by $\left(\mathbf{S}^{\mathrm{T}} \mathbf{\Sigma}^{-1} \mathbf{S}\right)^{-1}$ does. Comparing the uncertainties obtained using only the RO log-dry pressure profiles in $\mathbf{S}$ to those from using only the IR spectra, it is 
TABLE 1. Global mean all-sky OLR changes contributed by tropospheric temperature (Ttrop), stratospheric temperature (Tstrat), tropospheric water vapor (qtrop), stratospheric water vapor (qstrat), and high-level cloud (Chgh) in the doubled- $\mathrm{CO}_{2}$ experiment. Optimal detection results obtained from IR spectra, RO dry pressure profiles, and IR-RO jointly are compared to PRP-determined reference values ("Truth"). Unit: $\mathrm{W} \mathrm{m}^{-2}$.

\begin{tabular}{lrrcr}
\hline \hline \multirow{2}{*}{$\begin{array}{c}\text { OLR } \\
\text { change }\end{array}$} & $\begin{array}{c}\text { PRP } \\
\text { "Truth" }\end{array}$ & \multicolumn{3}{c}{ Optimal detection } \\
\cline { 3 - 5 } & & \multicolumn{1}{c}{ IR } & RO & IR + RO \\
\hline$\delta F_{\text {Ttrop }}$ & 9.78 & $9.83 \pm 1.11$ & $10.41 \pm 2.08$ & $9.87 \pm 0.98$ \\
$\delta F_{\text {Tstrat }}$ & -0.47 & $-0.46 \pm 0.19$ & $-0.56 \pm 0.25$ & $-0.47 \pm 0.11$ \\
$\delta F_{\text {qtrop }}$ & -4.99 & $-4.94 \pm 0.77$ & $-5.19 \pm 1.12$ & $-5.02 \pm 0.59$ \\
$\delta F_{\text {qstrat }}$ & -0.27 & $-0.26 \pm 0.13$ & $-4.50 \pm 10.41$ & $-0.25 \pm 0.13$ \\
$\delta F_{\text {Chgh }}$ & -1.18 & $-1.19 \pm 1.49$ & N/A & $-1.21 \pm 1.37$ \\
\hline
\end{tabular}

evident that the OLR changes determined from RO logdry pressure are of lower accuracy than those from IR spectra. However, when the two measurements are combined, the uncertainties are reduced to values noticeably less than what are given by either measurement alone for tropospheric temperature and water vapor and for stratospheric temperature. The reason for the improved accuracy is illustrated in Fig. 2. The ellipses illustrate the joint probability density function (PDF) of OLR changes contributed by tropospheric temperature and water vapor. The major axis of the uncertainty ellipse derived with only the IR spectrum in the fingerprint vector has a slope very close to -1 , indicating compensating errors between the feedbacks. Although larger in area, indicating larger uncertainty, the uncertainty ellipse derived with only the RO log-dry pressure in the fingerprint vector intersects the IR-only uncertainty ellipse and thus effectively limits the IR-RO joint estimates to a smaller (area reduced by $25 \%$ ) and less tilted (correlation reduced from 0.53 to $0.35)$ ellipse. The overall effect is to reduce the uncertainty in OD results and decrease the incidence of compensating errors. Since the information content of a measurement can be defined as the improvement of the knowledge of a quantity by making the measurement (Rodgers 2000), the result here signifies complementary information content of the IR and RO measurements. Although the RO measurement is insensitive to cloud, by reducing the uncertainty in tropospheric temperature, which is ambiguous with highlevel cloud (Huang et al. 2010), the uncertainty in the highcloud estimate is also reduced (see last row in Table 1).

Second, we use a set of global mean fingerprints $\mathbf{S}$ developed from global mean normalized partial change signals, the $i$ th column of $\mathbf{S}$ being $\mathbf{s}_{i}=\left[\left\langle\delta R_{i} / \delta F_{i}\right\rangle,\left\langle\left(\delta \ln P_{d}\right)_{i} /\right.\right.$ $\left.\delta F_{i}\right\rangle$ ] (see Fig. 3), and apply them to the total changes in the IR spectra and RO dry pressure profiles $\left[\delta R_{\text {total }}\right.$, $\left.\left(\delta \ln P_{d}\right)_{\text {total }}\right]$ at every GCM grid point. In this case, the performance of OD can be assessed by the globally averaged root-mean-square errors (RMSEs) in detected

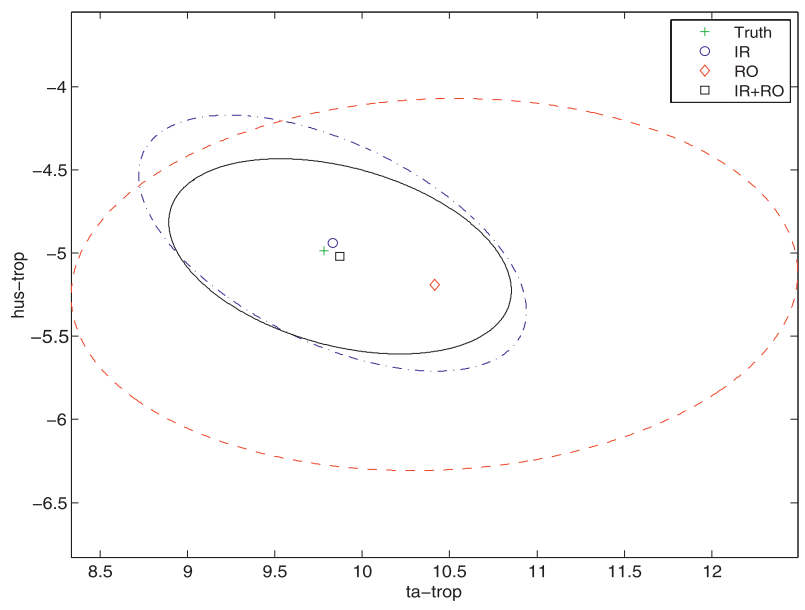

FIG. 2. Joint detection of global mean tropospheric temperature and water vapor contributions to OLR change. The small circles and ellipses illustrate the joint estimates and the PDFs of ( $\delta F_{\text {Ttrop }}$, $\left.\delta F_{\text {qtrop }}\right)$, optimally detected by IR only (blue), RO only (red), and combined IR + RO (black) measurements. The ellipses are drawn according to $\left(\mathbf{a}_{\mathrm{ml}}-\mathbf{a}\right)^{\mathrm{T}} \mathbf{S}^{\mathrm{T}} \mathbf{\Sigma}^{-1} \mathbf{S}\left(\mathbf{a}_{\mathrm{ml}}-\mathbf{a}\right)=1$; the accumulated probability within the ellipses equal $39 \%$ in the bivariate normal distribution case.

$\delta F_{i}$, which are given in Table 2. Comparing the IR-only detection and the IR-RO joint detection, it is evident that incorporating $\mathrm{RO}$ log-dry pressure considerably reduces the RMSEs in tropospheric and stratospheric temperature, tropospheric water vapor, and high cloud, generally by more than $50 \%$. From the global distribution of the biases (OD result minus truth; see Fig. 4), it is clear that the IR-only detection is subject to compensating errors in the ambiguous contributor pairs. Most noticeable are the offsetting errors in the Antarctic concerning tropospheric temperature and water vapor, and in the tropics concerning tropospheric temperature and high cloud. This also leads to large biases in the global mean of these detection results, which account for $43 \%, 20 \%$, and $31 \%$ in the total mean squared errors in tropospheric temperature, tropospheric water vapor, and high cloud, respectively. In the IR-RO joint detection, however, such compensating errors are largely suppressed. Unimproved, however, are the stratospheric water vapor and the surface temperature feedbacks, to which GNSS RO is insensitive.

The two tests show that adding RO log-dry pressure to the IR spectrum in the fingerprint of climate change noticeably improves the quantification of the longwave feedbacks of tropospheric temperature, tropospheric water vapor, stratospheric temperature, and high cloud. The GNSS RO measurement particularly helps resolve the ambiguity, when only IR measurement is used, between tropospheric temperature and tropospheric water vapor, and between tropospheric temperature and high cloud. Based on the results from test 2, the RMSE is reduced by 
6032

JOURNAL OF CLIMATE

VOLUME 23

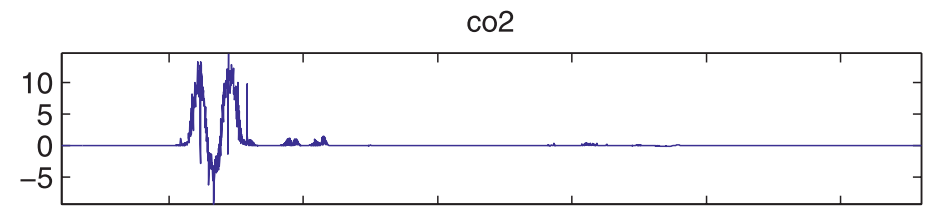

ts
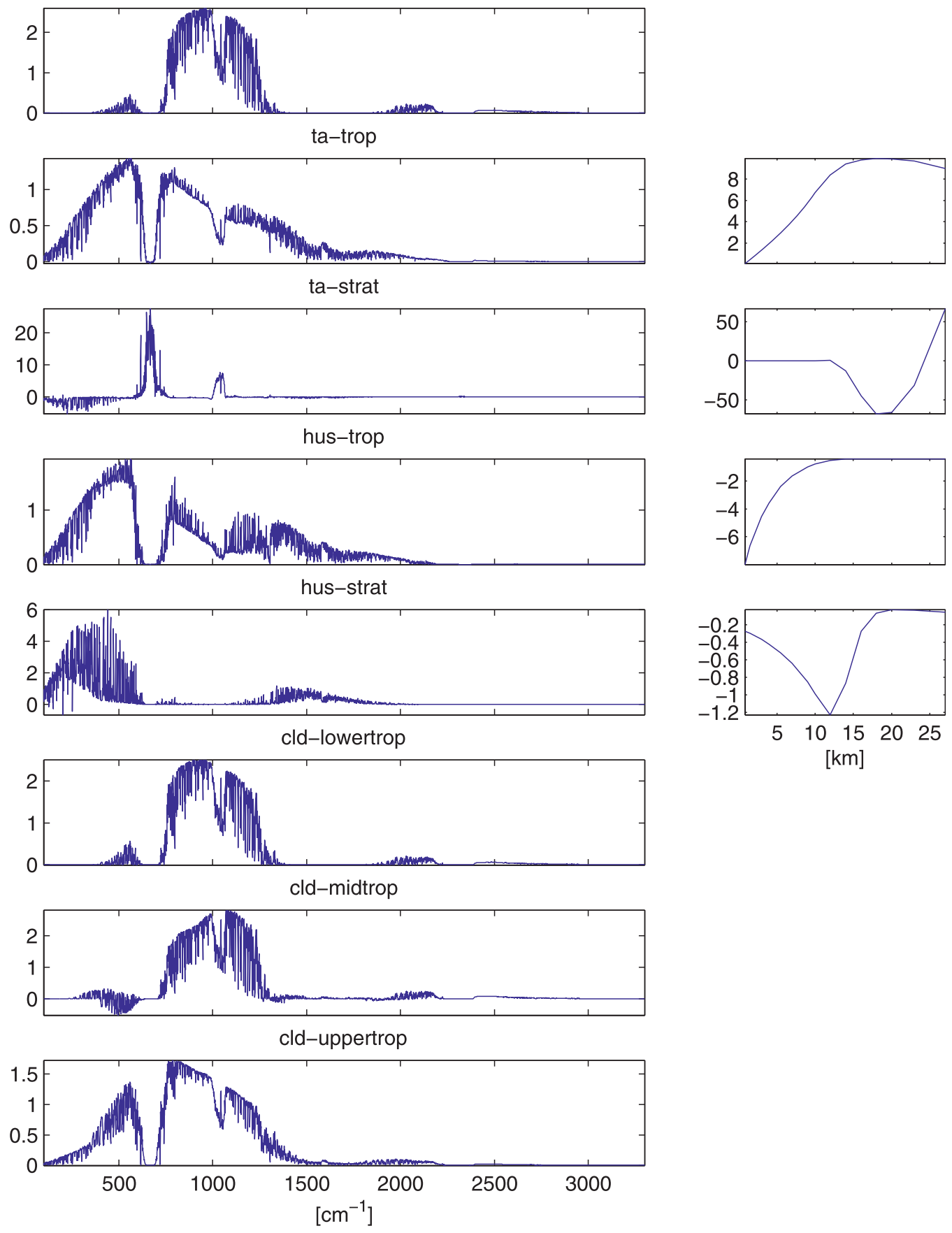

FIG. 3. Global mean IR spectral and GNSS RO log-dry pressure fingerprints. (left) Infrared spectral fingerprints for $\mathrm{CO}_{2}$ forcing, surface temperature change, and feedbacks in tropospheric temperature, stratospheric temperatore, tropospheric water vapor, stratospheric water vapor, and low, middle, and high clouds in order. (right) Four GNSS RO log-dry pressure fingerprints of the same feedbacks as the spectral fingerprints in the same row. 
TABLE 2. Global RMSE in optimally detected OLR changes. Unit: $\mathrm{W} \mathrm{m}^{-2}$.

\begin{tabular}{lccc}
\hline \hline & IR & RO & IR + RO \\
\hline$\delta$ OLR $_{\text {co2 }}$ & 0.10 & N/A & 0.11 \\
$\delta$ OLR $_{\text {Ts }}$ & 3.27 & N/A & 3.88 \\
$\delta$ OLR $_{\text {Ttrop }}$ & 2.02 & 2.36 & 0.98 \\
$\delta$ OLR $_{\text {Tstrat }}$ & 0.25 & 0.14 & 0.09 \\
$\delta$ OLR $_{\mathrm{qtrop}}$ & 1.32 & 1.47 & 0.73 \\
$\delta$ OLR $_{\mathrm{qstrat}}$ & 0.10 & 6.36 & 0.12 \\
$\delta \mathrm{OLR}_{\text {Clow }}$ & 3.76 & $\mathrm{~N} / \mathrm{A}$ & 3.66 \\
$\delta$ OLR $_{\text {Cmid }}$ & 1.36 & $\mathrm{~N} / \mathrm{A}$ & 1.25 \\
$\delta$ OLR $_{\text {Chgh }}$ & 3.05 & $\mathrm{~N} / \mathrm{A}$ & 1.07 \\
\hline
\end{tabular}

$51 \%$ for tropospheric temperature feedback, $64 \%$ for stratospheric temperature feedback, $45 \%$ for tropospheric water vapor feedback, and $65 \%$ for high cloud feedback. The remaining RMSEs relative to their global mean feedback values are $4 \%$ for $\mathrm{CO}_{2}$ forcing, $10 \%$ for tropospheric temperature feedback, $15 \%$ for tropospheric water vapor feedback, $20 \%$ for stratospheric temperature feedback, $44 \%$ for stratospheric water vapor feedback, $91 \%$ for high cloud feedback, and $115 \%$ for surface temperature response; they are much larger for low and middle cloud feedbacks.

\section{Discussion and conclusions}

We have explored how to quantitatively evaluate longwave climate forcing and feedbacks from multiple measurements by using an optimal detection method. Compared to the thousands of infrared radiance channels that are typical of a spaceborne spectrometer, the information content in the GNSS RO dry pressure profiles of merely 17 or so vertical levels seems very small. Indeed, if the two measurements are used to quantify the longwave climate feedback independently, the uncertainty in longwave feedbacks derived using only RO log-dry pressure is generally larger than that using spectral measurement. However, the climate change signal in the GNSS RO log-dry pressure profile induced by atmospheric temperature is distinct from that induced by water vapor or cloud, so that the ambiguity issue that affects the linear partitioning of these feedbacks in the IR spectrum does not impede the partitioning in the $\mathrm{RO} \log$ dry pressure profile. We have demonstrated that when the two measurements are jointly used to quantify the feedbacks, the additional information provided by the GNSS RO measurement can be critically helpful to improve the accuracy in the results.

Because we have applied optimal detection to synthetic IR spectra and log-dry pressure profiles, much as in Huang et al. (2010), the accuracy in the feedback detection results estimated in this study may quantitatively differ from what is associated with real data.
However, our estimate of the uncertainty is likely a conservative one in terms of global mean feedback values because the fingerprint uncertainty, a key source of the uncertainty in the feedbacks, is measured by the variation of the fingerprints over all the GCM grid points (all the samples allowed by the doubled- $\mathrm{CO}_{2}$ experiment). Because the variation of the climate states of different regions in the GCM exceeds the uncertainty in our best knowledge of the present global mean climate state and the variation of the changes in the climate states of different regions in the GCM also likely exceeds the uncertainty in the change of global mean climate state, the uncertainty we estimate is most likely an over-estimate of the actual uncertainty.

With three sources of uncertainty (fingerprint uncertainty, natural variability, and nonlinearity) considered, we show that the global mean values in $\mathrm{CO}_{2}$ forcing and longwave feedbacks of tropospheric temperature, tropospheric water vapor, and stratospheric temperature can be accurately determined from IR spectral and GNSS RO measurements, with RMSEs in them generally being less than $20 \%$. Because of unresolved ambiguities, however, the accuracy in surface temperature response and cloud feedbacks remains poor, but it can likely be improved by incorporating other types of measurement. For instance, reflected solar radiances may resolve the IR ambiguity between low cloud and surface temperature changes over most types of surfaces. Note, however, that combining another data type may introduce new ambiguities as well. In the reflected solar radiation case, an ambiguity may occur between cloud and ice-covered surface or between cloud and aerosol, which IR spectral measurement may resolve to a certain extent in turn. Further investigations should reveal whether and by how much the addition of a shortwave radiation data type can resolve the low cloud ambiguity.

This study demonstrates that the optimal detection method is suitable for integrating the infrared spectral and radio occultation measurements in climate feedback analysis. This methodology can be applied to the climatic datasets that are to be made available by the existing and planned spaceborne infrared spectral and radio occultation measurements such as the Atmospheric Infrared Sounder (AIRS) on the Aqua satellite, the Infrared Atmospheric Sounding Interferometer (IASI) on MetOp, the Crosstrack Infrared Sounder (CrIS) planned for the National Polar-Orbiting Operational Environmental Satellite System (NPOESS), the Constellation Observing System for Meteorology Ionosphere and Climate (COSMIC), and the planned Climate Absolute Radiance and Refractivity Observatory (CLARREO). Given the large feedback discrepancies in the present climate models (Bony et al. 2006; Solomon et al. 2007), such observational 

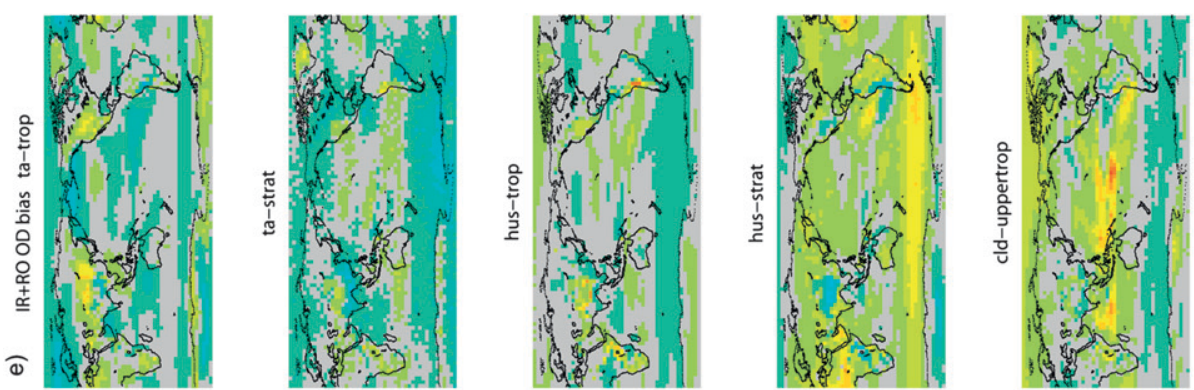

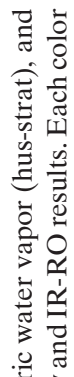
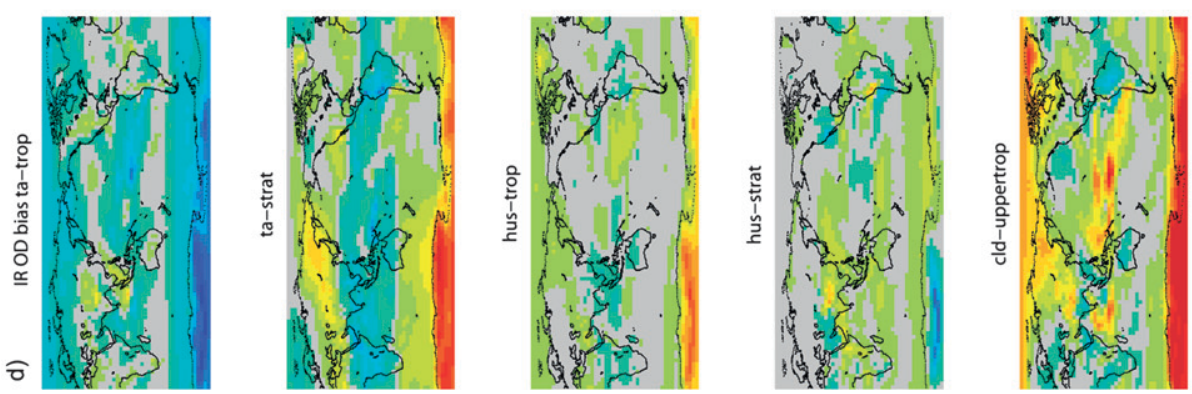

름

원

:

产

के

ग

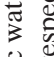

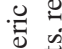
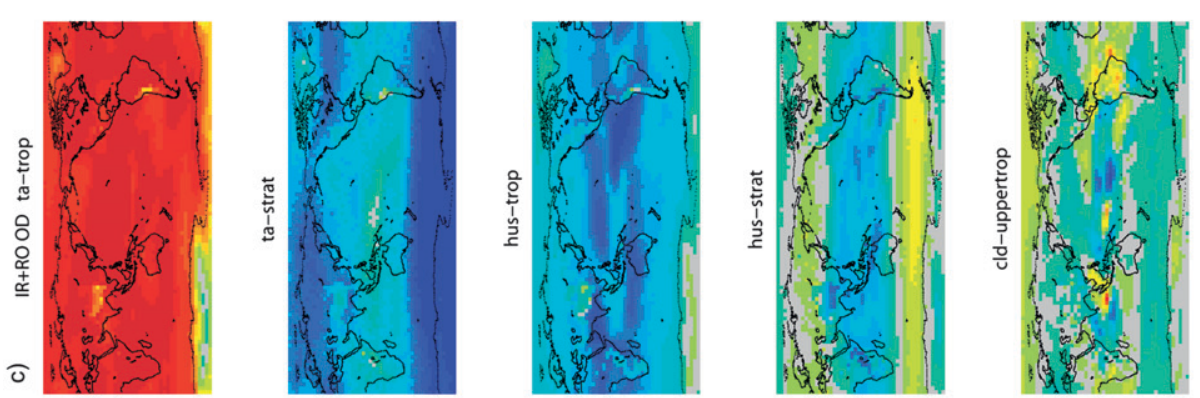

包高
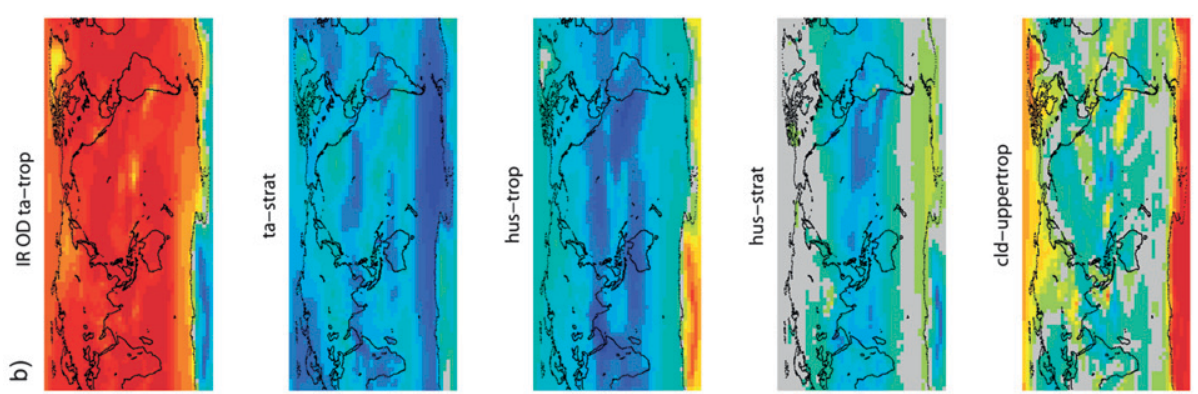

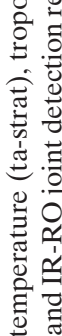

冚

तै

额

के

किष

훌

害

可

害吕言

농 总
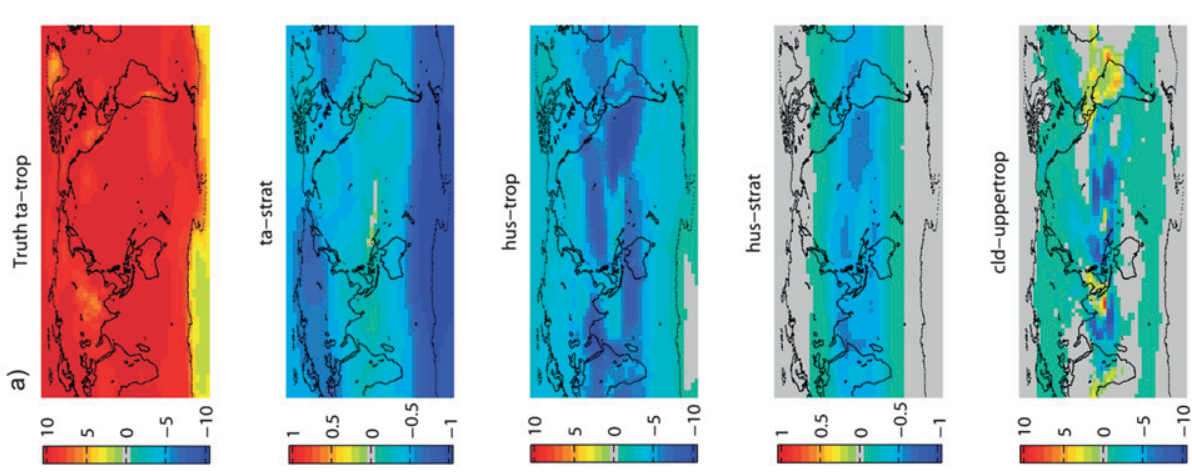

흥믈

의의

$\circ \frac{1}{0}$

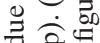

㻤

층 층

$\therefore$ 定

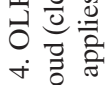

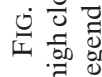


data-based analysis will provide strong constraints on the feedback values and climate sensitivity and help discriminate climate models in terms of decadal climate change projection. When real observational data are used for detecting the feedbacks, a rigorous estimate of the overall uncertainty will require accounting for instrumental noise and spatiotemporal sampling error, as well as an appropriate quantification of natural variability and fingerprint uncertainty. For example, simultaneous IR and $\mathrm{RO}$ soundings of the same object rarely exist due to their different remote sensing techniques (nadir versus occultation), so the joint fingerprinting can only be based on contemporaneous data. The constraint of spatial and temporal sampling and averaging needs to be assessed with specific instrument orbits and measurement rates. Meanwhile, it is important to understand how long it takes the measurement types to constrain the transient climate sensitivity. When applying the method to transient climate change signals, at decadal scale, the uncertainty due to natural variability can be assessed from a simulated multidecadal time series; and the fingerprint uncertainty can be derived from a multi-GCM ensemble. In the doubled- $\mathrm{CO}_{2}$ experiment, fingerprint uncertainty is the dominant source of uncertainty, whereas in an application to short time scales, natural variability may dominate over fingerprint uncertainty. These various aspects should be investigated in future studies in accordance with a realistic instrumental model.

Acknowledgments. We thank three anonymous reviewers who helped improve this paper. Yi Huang is supported by a NOAA Climate and Global Change postdoctoral fellowship.

\section{REFERENCES}

Bell, T., 1986: Theory of optimal weighting of data to detect climate change. J. Atmos. Sci., 43, 1694-1710.

Bernstein, L., A. Berk, P. Acharya, D. Robertson, G. Anderson, J. Chetwynd, and L. Kimball, 1996: Very narrow band model calculations of atmospheric fluxes and cooling rate. J. Atmos. Sci., 53, 2887-2904.

Bony, S., and Coauthors, 2006: How well do we understand and evaluate climate change feedback processes? J. Climate, 19, 3445-3482.

Goody, R., J. Anderson, and G. North, 1998: Testing climate models: An approach. Bull. Amer. Meteor. Soc., 79, 2541-2549.

Haskins, R., R. Goody, and L. Chen, 1999: Radiance covariance and climate models. J. Climate, 12, 1409-1422.
Hasselmann, K., 1997: Multi-pattern fingerprint method for detection and attribution of climate change. Climate Dyn., 13, 601-611.

Huang, Y., and V. Ramaswamy, 2009: Evolution and trend of outgoing longwave radiation spectrum. J. Climate, 22, 46374651.

,,-- X. Huang, Q. Fu, and C. Bardeen, 2007a: A strict test in climate modeling with spectrally resolved radiances: GCM simulation versus AIRS observations. Geophys. Res. Lett., 34, L24707, doi:10.1029/2007GL031409.

, and B. Soden, 2007b: An investigation of the sensitivity of the clear-sky outgoing longwave radiation to atmospheric temperature and water vapor. J. Geophys. Res., 112, D05104, doi:10.1029/2005JD006906.

- S. Leroy, P. Gero, J. Dykema, and J. Anderson, 2010: Separation of longwave climate feedbacks from spectral observations. J. Geophys. Res., 115, D07104, doi:10.1029/ 2009JD012766.

Kiehl, J., 1983: Satellite detection of effects due to increased atmospheric carbon dioxide. Science, 222, 504-506.

Kursinski, E., G. Hajj, J. Schofield, R. Linfield, and K. Hardy, 1997: Observing Earth's atmosphere with radio occultation measurements using the Global Positioning System. J. Geophys. Res., 102 (D19), 23 429-23 465.

Leroy, S., 1997: Measurement of geopotential heights by GPS radio occultation. J. Geophys. Res., 102 (D6), 6971-6986. , and G. North, 2000: The application of COSMIC data to global change research. Terr. Atmos. Ocean. Sci., 11, 187-210. and J. Anderson, 2010: Optimal detection of regional trends using global data. J. Climate, 23, 4438-4446.

,-- , and J. Dykema, 2006: Testing climate models using GPS radio occultation: A sensitivity analysis. J. Geophys. Res., 111, D17105, doi:10.1029/2005JD006145.

,,$-- \ldots$, and R. Goody, 2008: Testing climate models using thermal infrared spectra. J. Climate, 21, 1863-1875.

McClatchey, R., R. Fenn, J. Selby, R. Voltz, and J. Garing, 1972: Optical Properties of the Atmosphere. Air Force Cambridge Research Laboratories, 108 pp.

North, G., K. Kim, S. Shen, and J. Hardin, 1995: Detection of forced climate signals. I: Filter theory. J. Climate, 8, 401-408.

Rodgers, C., 2000: Inverse Methods for Atmospheric Sounding: Theory and Practice. World Scientific, $238 \mathrm{pp}$.

Slingo, A., and M. Webb, 1997: The spectral signature of global warming. Quart. J. Roy. Meteor. Soc., 123, 293-307.

Smith, E., and S. Weintraub, 1953: The constants in the equation for atmospheric refractive index at radio frequencies. Proc. IEEE, 41, 1035-1037.

Solomon, S., D. Qin, M. Manning, M. Marquis, K. Averyt, M. M. B. Tignor, H. L. Miller Jr., and Z. Chen, Eds., 2007: Climate Chance 2007: The Physical Science Basis. Cambridge University Press, 996 pp.

Wetherald, R., and S. Manabe, 1988: Cloud feedback processes in a general circulation model. J. Atmos. Sci., 45, 1397-1415.

Williams, K., and M. Webb, 2009: A quantitative performance assessment of cloud regimes in climate models. Climate Dyn., 33, 141-157. 